\title{
Interaction of Luminescent Defects in Carbon Nanotubes with Covalently Attached Stable Organic Radicals
}

\author{
Felix J. Berger, J. Alejandro de Sousa, Shen Zhao, Nicolas F. Zorn, Abdurrahman Ali El Yumin, \\ Aleix Quintana García, Simon Settele, Alexander Högele, Núria Crivillers, and Jana Zaumseil*
}

Cite This: ACS Nano 2021, 15, 5147-5157

Read Online

ACCESS | 岁 Metrics \& More | 回 Article Recommendations | S1 Supporting Information

ABSTRACT: The functionalization of single-walled carbon nanotubes (SWCNTs) with luminescent $\mathbf{s p}^{3}$ defects has greatly improved their performance in applications such as quantum light sources and bioimaging. Here, we report the covalent functionalization of purified semiconducting SWCNTs with stable organic radicals (perchlorotriphenylmethyl, PTM) carrying a net spin. This model system allows us to use the near-infrared photoluminescence arising from the defectlocalized exciton as a highly sensitive probe for the shortrange interaction between the PTM radical and the SWCNT.

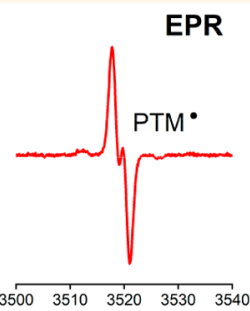

Magnetic field $(G)$
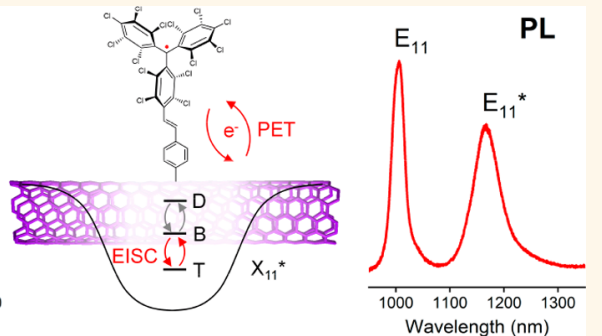

Our results point toward an increased triplet exciton population due to radical-enhanced intersystem crossing, which could provide access to the elusive triplet manifold in SWCNTs. Furthermore, this simple synthetic route to spin-labeled defects could enable magnetic resonance studies complementary to in vivo fluorescence imaging with functionalized SWCNTs and facilitate the scalable fabrication of spintronic devices with magnetically switchable charge transport.

KEYWORDS: single-walled carbon nanotube, $s p^{3}$ defect, stable organic radical, photoluminescence, triplet, magnetic field

$\mathrm{T}$ he controlled covalent modification of single-walled carbon nanotubes (SWCNTs) has boosted their potential for a broad range of applications including quantum light emission, ${ }^{1}$ bioimaging, ${ }^{2}$ and sensing. ${ }^{3}$ The onedimensional structure of SWCNTs, their large charge carrier mobilities, ${ }^{4}$ and high exciton diffusivity ${ }^{5}$ make them ideal for efficient interaction of charges and excitations with lattice defects, thus further expanding the nanotubes' properties. In recent years, the low-density functionalization of semiconducting SWCNTs with luminescent $\mathrm{sp}^{3}$ defects, sometimes referred to as organic color centers or quantum defects, has received particular attention. ${ }^{6-8}$ The deep optical trap potentials $(\sim 100$ $\mathrm{meV}$ ) of these defects created by binding of oxygen, ${ }^{9}$ aryl, ${ }^{10}$ or alkyl $^{11}$ substituents lead to efficient exciton localization. These trapped excitons give rise to red-shifted photoluminescence (PL) with long lifetimes and even enable room-temperature single-photon emission at telecommunication wavelengths. ${ }^{12}$ As the defect states arise from the perturbation of the SWCNTs' electronic structure, their properties are dominated by the precise binding pattern with respect to the SWCNT lattice rather than the molecular structure of the substituents. ${ }^{13}$ Hence, the molecular groups that are bound to the nanotube surface are often structurally simple, and only few examples are known, in which the attached group contributes and performs a function itself, such as sensing ${ }^{14-16}$ or anchoring of biomolecules. $^{17}$

A highly desirable property for functional groups attached to SWCNTs is a net spin, as carried by unpaired electrons. Previously, mixed-chirality samples of SWCNTs and multiwalled carbon nanotubes were decorated with open-shell transition metal ${ }^{18}$ or lanthanide ${ }^{19,20}$ complexes, and charge transport measurements at cryogenic temperatures revealed spin valve switching behavior without the need for ferromagnetic contacts. ${ }^{19,20}$ Another class of spin-bearing compounds are stable organic radicals, i.e., charge-neutral molecules with an unpaired electron. ${ }^{21,22}$ One of the most prominent examples is the perchlorinated triphenylmethyl (PTM) radical, in which a propeller-shaped arrangement of phenyl rings protects the carbon-centered radical, resulting in a half-life of $\sim 100$ years. ${ }^{23}$ The interaction of SWCNTs with the

Received: December 10, 2020

Accepted: February 5, 2021

Published: February 18, 2021 


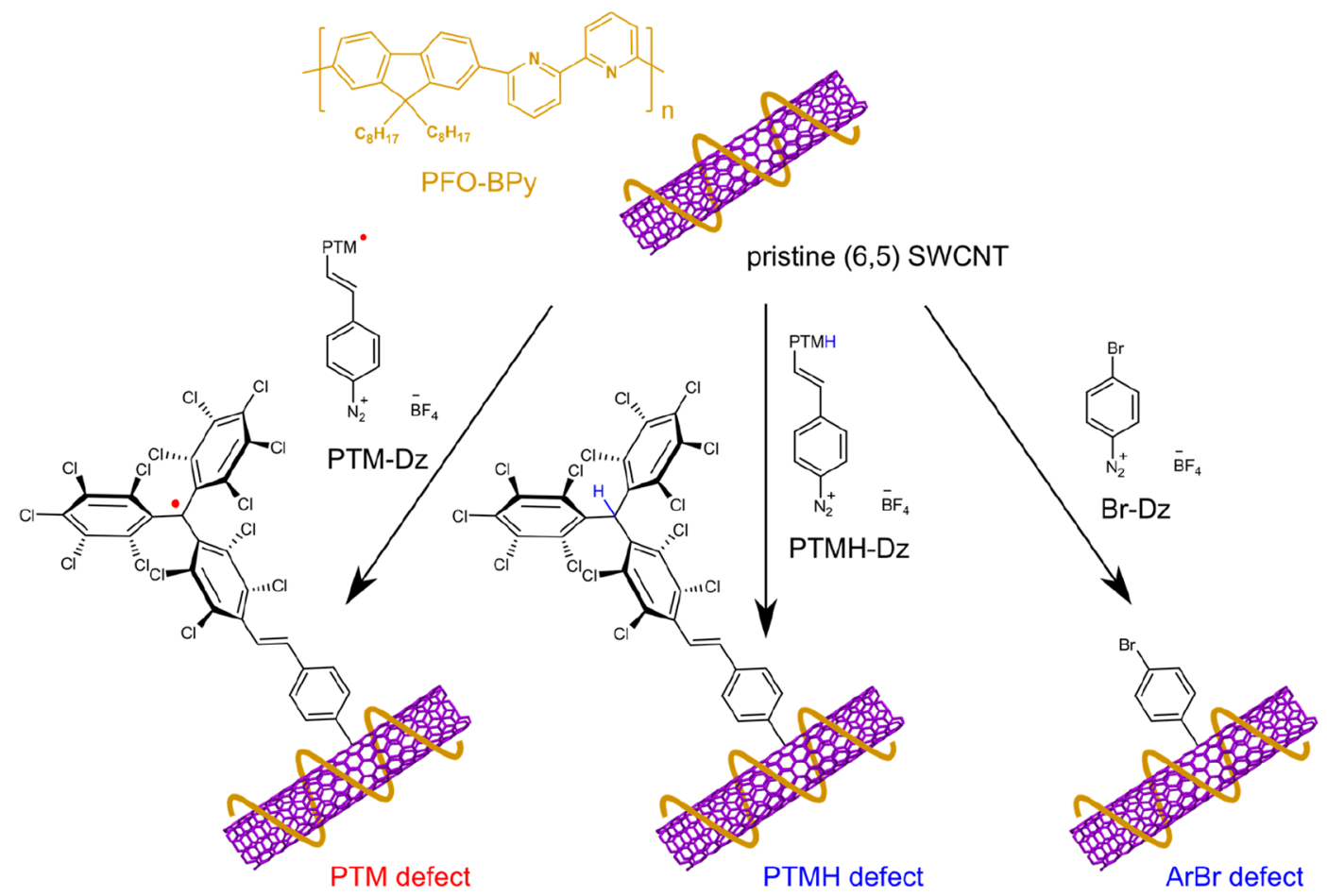

Figure 1. Reaction scheme depicting the functionalization of polymer-wrapped (6,5) SWCNTs with aryl defects bearing radical (PTM, red) or closed-shell (PTMH, ArBr, blue) substituents.

a)

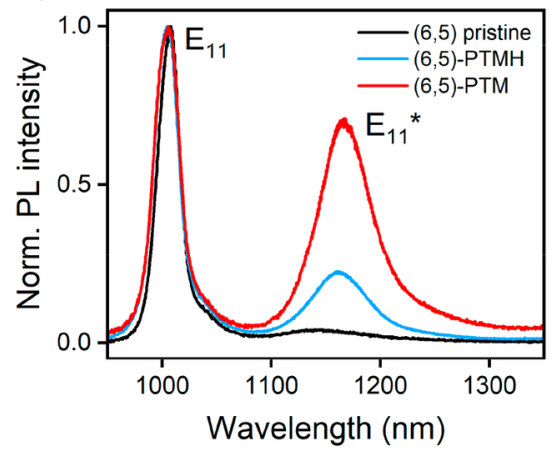

b)

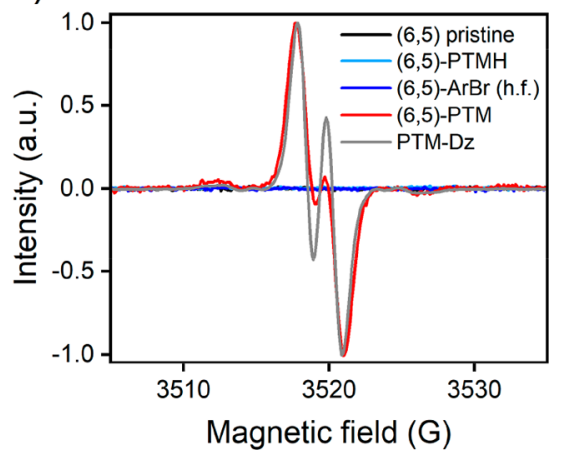

Figure 2. (a) Normalized PL spectra recorded for dispersions of pristine and PTM- and PTMH-functionalized (6,5) SWCNTs in toluene. (b) EPR spectra (at room temperature) of these dispersions in addition to a dispersion of $(6,5)$ SWCNTs with a high density of ArBr defects (h.f.) and a solution of the PTM-Dz precursor in tetrahydrofuran.

unpaired spin of the radical via a luminescent $\mathrm{sp}^{3}$ defect may result in spin-dependent properties and provide optical means for probing them. However, up to now there are no reports of stable organic radicals bound to carbon nanotubes or chiralitysorted SWCNTs with any covalent spin label.

In this work, we functionalize purely semiconducting $(6,5)$ SWCNTs with aryl defects linked to PTM radicals and use the PL arising from the defect-localized exciton as a highly sensitive probe for the short-range interaction between the radical and the SWCNT. By comparing them to aryl defects with closed-shell substituents, we observe PL quenching associated with the radical. Based on the PL decay dynamics, we propose partial transfer of the exciton population to triplet states via radical-enhanced intersystem crossing as the dominant quenching mechanism, while a smaller portion may be attributed to a photoinduced electron transfer process. In addition to providing a simple route to spin-labeled defects for magnetic resonance studies and spintronics, radical functionalization could improve the accessibility of the elusive triplet states in carbon nanotubes.

\section{RESULTS AND DISCUSSION}

SWCNT Functionalization and Characterization. Highly purified $(6,5)$ SWCNTs (diameter $\sim 0.76 \mathrm{~nm}$, average length $1.0-1.5 \mu \mathrm{m}$ ) wrapped with the fluorene-bipyridine copolymer PFO-BPy (see the Methods and Figure 1 for molecular structure $)^{24}$ serve as a robust model system for $\mathrm{sp}^{3}$ functionalization. As shown in Figure 1, diazonium $(\mathrm{Dz})$ chemistry was used to introduce aryl defects bearing chargeneutral substituents with either radical (open-shell) or closedshell character. Reacting (6,5) SWCNTs with the tailored diazonium salt PTM-Dz ${ }^{25,26}$ produced aryl defects on the nanotube lattice that act as covalent linkers to PTM radicals. To identify the impact of the radical on defect state PL, the 
a)

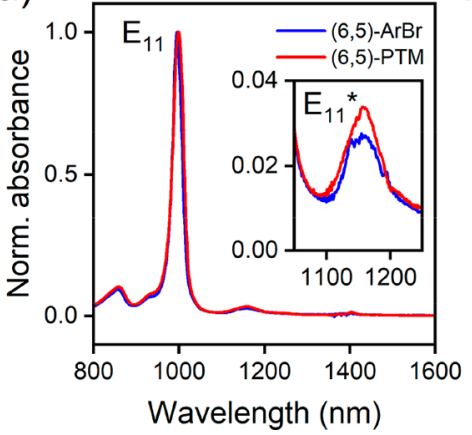

b)

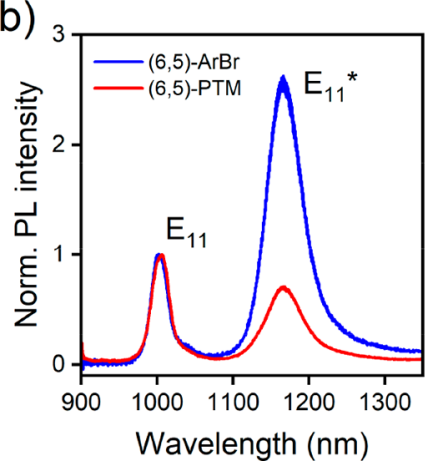

c)

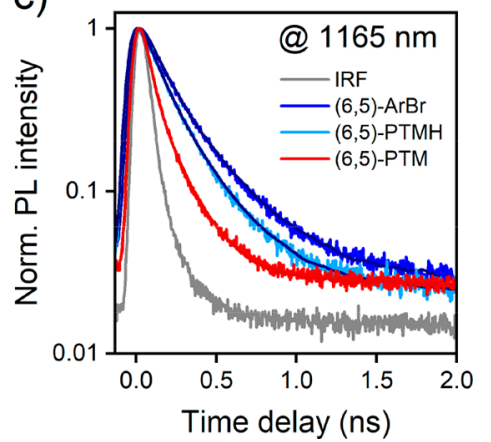

Figure 3. (a) Absorption and (b) PL spectra of PTM- and ArBr-functionalized (6,5) SWCNTs with similar defect density. (c) PL decay traces of defect emission at $1165 \mathrm{~nm}$. All data were measured on SWCNT dispersions in toluene.

closed-shell hydrogen-substituted PTMH-Dz was employed to create reference defects. Additionally, simple closed-shell bromoaryl defects $(\mathrm{ArBr})$ were produced by reaction with the commercial $\mathrm{Br}-\mathrm{Dz}$ reagent, which exhibited a higher reactivity than the PTMH-Dz and was therefore used for experiments requiring larger defect densities. Since polymerwrapped SWCNTs form stable dispersions only in low-polarity solvents, the functionalization with $\mathrm{Br}-\mathrm{Dz}$ was facilitated by ether crown complexation in a toluene/acetonitrile mixture following our recently reported protocol. ${ }^{27}$ In contrast to that, PTM-Dz and PTMH-Dz are well-soluble in tetrahydrofuran (THF), which also disperses PFO-BPy-wrapped SWCNTs. They are therefore ideally suited for the reaction with polymerwrapped SWCNTs without the need for any solubilizing agents. Nanotube functionalization was always performed in the dark and completed within $16 \mathrm{~h}$ at room temperature. Subsequently, the functionalized SWCNTs were collected by vacuum filtration, carefully washed with pure solvent to remove unreacted diazonium salt and byproducts, and finally redispersed in fresh toluene for characterization.

To confirm the covalent functionalization of $(6,5)$ SWCNTs with PTM radicals, photoluminescence and electron paramagnetic resonance (EPR) spectra were recorded for toluene dispersions of the nanotubes at room temperature (Figure 2). Here, we focus on a comparison between PTM radicalfunctionalized SWCNTs and the corresponding closed-shell PTMH-functionalized SWCNTs as well as pristine SWCNTs. All $(6,5)$ SWCNT dispersions exhibit photoluminescence at $1006 \mathrm{~nm}$ originating from mobile excitons recombining on pristine lattice segments ( $\mathrm{E}_{11}$ transition). In addition, both $\mathrm{sp}^{3}$ functionalized nanotubes show emission at $\sim 1165 \mathrm{~nm}$, which is characteristic of defect-localized excitons $\left(\mathrm{E}_{11} *\right)$ and strong evidence for the covalent attachment of molecular groups to the nanotube lattice (Figure 2a). To analyze the influence of the radical substituent on the defect state energy, the optical trap depth ${ }^{28}\left(E_{11}-E_{11} *\right)$ was extracted from the PL spectra of PTM- and PTMH-functionalized SWCNTs (Supporting Information Figure S1). The optical trap depth of PTM defects $(172 \mathrm{meV})$ was slightly larger than that of PTMH defects $(168 \mathrm{meV})$, which is in agreement with the stronger electron-withdrawing character of the PTM group. ${ }^{10}$ However, the difference is small, presumably due to the relatively long distance between the central carbon of the PTM moiety and the SWCNT lattice. We can conclude that the optical trap depth should only play a minor role with regard to any differences observed between PTM and PTMH defects.
While the presence of an $\mathrm{E}_{11}$ * emission feature clearly indicates covalent functionalization, it is relatively insensitive to the precise molecular structure of the attached group. ${ }^{13}$ Hence, EPR measurements were performed to ascertain that the substituent was indeed the desired PTM-styryl unit and the radical character was preserved after the reaction. Figure $2 \mathrm{~b}$ shows that the pristine $(6,5)$ SWCNTs do not display any EPR signal, as expected for highly purified semiconducting SWCNTs. ${ }^{29}$ Likewise, closed-shell PTMH-functionalized SWCNTs show no EPR intensity at room temperature. To make sure that this result was not compromised by the low density of PTMH defects, a second control experiment was performed on $(6,5)$ SWCNTs that were highly functionalized with closed-shell $\mathrm{ArBr}$ defects, which also did not give rise to any EPR signal (see Supporting Information Figure S2 for additional characterization). The PTM-functionalized SWCNTs, on the other hand, show the clear EPR signature of the PTM-styryl unit with a $g$ factor of 2.0024, which is characteristic for PTM radicals, and a peak splitting due to hyperfine coupling between the PTM radical and the vinylene proton with appropriate symmetry (for more details on the fine structure see the Supporting Information Figure S3). Furthermore, the EPR signal of the PTM radical attached to the SWCNT is broadened compared to that of the molecular precursor PTM-Dz (line widths of 1.4 and $1.1 \mathrm{G}$, respectively; see Supporting Information Figure S4). This broadening may be attributed to the slow tumbling ${ }^{30}$ of the micrometer-long nanotube in dispersion with a rotational correlation time of $\sim 10 \mathrm{~ms} \mathrm{rad}^{-1}$, which is much longer than that of the unbound PTM-Dz molecule. Consequently, the line broadening further corroborates the successful decoration of SWCNTs with stable organic radicals.

These results clearly indicate that the room-temperature EPR response of PTM-tailored SWCNTs originates from an unpaired spin localized on the PTM moiety and not on the nanotube itself. In contrast to that, Lohmann et al. ${ }^{32}$ recently reported that aryl-functionalization of SWCNTs with closedshell substituents in aqueous dispersions may give rise to an EPR signal at low temperatures. The authors assigned this signal to a nanotube-centered radical, which might be formed upon addition of an odd number of $\mathrm{sp}^{3}$ defects to the lattice. Since the intensity of this EPR signal decreased strongly with increasing temperature and became undetectable above 200 $\mathrm{K},{ }^{32}$ such a feature is not expected to appear in our roomtemperature EPR measurements.

Impact of Radical on Defect PL. The unusual observation of a pronounced absorption band accompanied 
by relatively weak PL from the PTM defects (compare Figure 2 and Supporting Information Figure S2) prompted a more detailed investigation of their PL efficiency in relation to a reference sample of closed-shell ArBr-functionalized SWCNTs with similar defect density, as indicated by the $\mathrm{E}_{11} * / \mathrm{E}_{11}$ absorbance ratio (Figure 3a). As shown in Figure $3 b$, the radical-functionalized defects exhibit a significantly lower $\mathrm{E}_{11} * /$ $\mathrm{E}_{11}$ PL intensity ratio than the closed-shell reference despite having a similar defect density. This picture is complemented by PL lifetime measurements via time-correlated single-photon counting (TCSPC) for the $\mathrm{E}_{11} *$ PL from different defect types. The data indicate a substantially shorter decay time for the radical-functionalized defects compared to both closed-shell references (Figure 3c). It should be noted that the emission from all samples was filtered by a grating spectrograph to pass only the intensity from a narrow wavelength band around 1165 $\mathrm{nm}$ to the single-photon detector. Thereby, we rule out the wavelength-dependence of defect state lifetime as a possible cause for the observed differences. ${ }^{33}$ Combined with the reduced defect emission intensity, the shortened PL lifetime of PTM-functionalized SWCNTs points toward a nonradiative relaxation pathway associated with the radical in close proximity to the defect. Notably, the $\mathrm{E}_{11}$ * PL decay of the radical-functionalized SWCNTs can still be described by the biexponential model typical for localized exciton decay dynamics at room temperature. ${ }^{33,34}$

If the observed $\mathrm{E}_{11} * \mathrm{PL}$ quenching results from the presence of the radical substituent on the aryl defect, the in situ conversion of open-shell to closed-shell substituents on the $\mathrm{sp}^{3}$ defects will be the most direct way to confirm and quantify this effect. For this purpose, we exploited the fact that PTM radicals are sensitive to UV and blue light (in the range of their absorption maximum around $385 \mathrm{~nm}$ ). It is well-established that irradiation in this spectral range induces chlorine atom elimination from the PTM followed by ring closure to the perchlorophenylfluorenyl (PPF) radical. ${ }^{35,36}$ Due to the planar structure of the fluorene unit hosting the radical, the PPF radical is much more susceptible to chemical attack than the PTM radical. Hence, we consider hydrogen atom abstraction from the solvent and formation of the closed-shell PPFH species as a plausible decomposition pathway, as shown schematically in Supporting Information Figure S5. In practice, degradation of the PPF radical yields a mixture of closed-shell products. Although the extremely low defect concentrations (estimated to be in the range of $\mathrm{nmol} / \mathrm{L}$ ) did not allow us to determine the molecular structure of any photolysis products, we confirmed experimentally that all of them were of closedshell nature (see below). As noted in the discussion of optical trap depths of PTM versus PTMH defects, minor changes in substituent molecular structure far away from the SWCNT do not impact the $\mathrm{E}_{11} * \mathrm{PL}$ properties significantly; ${ }^{13}$ thus the $\mathrm{PPFH}$ substituent is a good closed-shell reference system for the open-shell PTM.

For in situ conversion, a dispersion of PTM-functionalized SWCNTs was exposed to UV light $\left(365 \mathrm{~nm}, 0.2 \mathrm{~W} \mathrm{~cm}^{-2}\right)$ for $40 \mathrm{~min}$ and PL spectra as well as PL decay traces were recorded under identical conditions before and after irradiation. As expected, the $\mathrm{E}_{11} * \mathrm{PL}$ intensity strongly increased due to light-induced radical degradation, whereas the $\mathrm{E}_{11}$ intensity remained constant (Figure $4 \mathrm{a}$, note that absolute PL intensities are compared). Hence, the creation of additional $\mathrm{sp}^{3}$ defects as the origin of increased $\mathrm{E}_{11}$ * intensity can be ruled out. In that case, the $E_{11}$ intensity would be a)

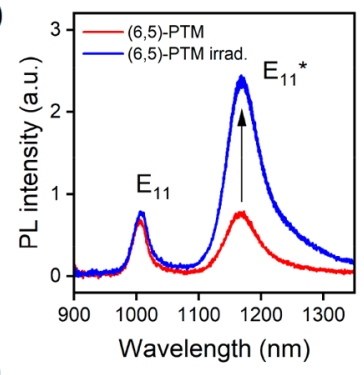

c)

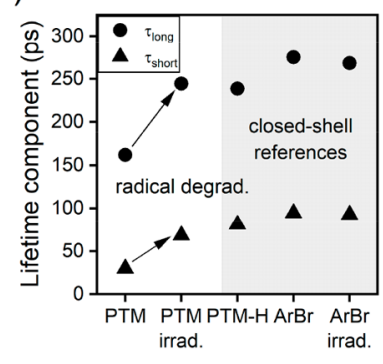

b)

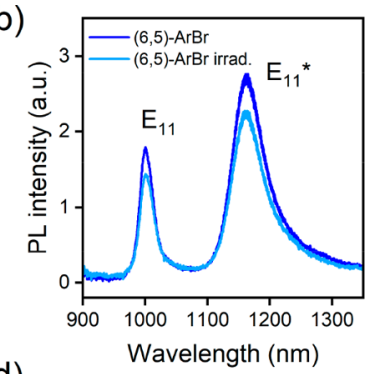

d)

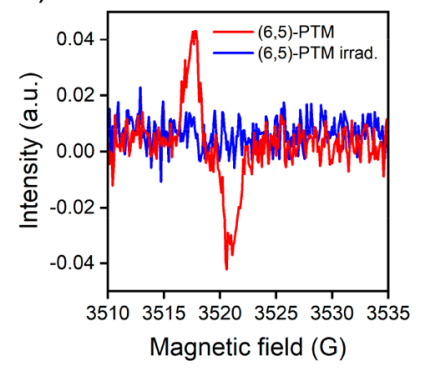

Figure 4. (a) PL spectra of PTM-functionalized and (b) ArBrfunctionalized $(6,5)$ SWCNTs before and after irradiation. (c) PL lifetime components extracted from biexponential fits to the decay traces. (d) EPR spectra recorded for the dispersions used in the PL experiments before and after irradiation. Note that the initial EPR signal intensity is relatively low, because dilute dispersions were employed for the optical characterization.

reduced due to the higher density of exciton trapping sites. The unchanged $\mathrm{E}_{11}$ emission confirms that the interaction between the radical and the SWCNT is confined to the $\mathrm{sp}^{3}$ defect. To exclude that these changes are related to photochemical reactions on the SWCNT lattice itself, e.g., rearrangement of $\mathrm{sp}^{3}$ defects, the experiment was repeated with a dispersion of $\mathrm{ArBr}$-functionalized SWCNTs. As shown in Figure $4 \mathrm{~b}$, these defects did not respond to UV irradiation. The minor drop of PL intensity is likely due to slight SWCNT aggregation over the course of irradiation, and the normalized spectra in Supporting Information Figure S6 illustrate that the PL spectral shape does not change at all. Hence, the $\mathrm{E}_{11} *$ brightening observed in Figure $4 \mathrm{a}$ can be attributed unambiguously to the photochemical transformation of the PTM radical to a closed-shell system.

The marked increase in $\mathrm{E}_{11}$ * emission efficiency can be understood by considering the prolonged PL lifetime of the defect-localized exciton following the open-shell to closed-shell conversion (Figure 4c). Upon irradiation, the initially short PL lifetime of the PTM defects rises to the level of the closed-shell references (PTMH and ArBr). By comparing EPR spectra of the PTM-functionalized SWCNT dispersion before and after irradiation (Figure 4d), we can conclude that the PTM radical was indeed converted to a closed-shell species. Consequently, the mechanism responsible for $\mathrm{E}_{11} * \mathrm{PL}$ quenching in PTMfunctionalized SWCNTs must be directly linked to the presence of the radical on the substituent of the aryl defect. The PL quenching effect can be quantified based on the PL spectral and lifetime data. Table 1 details the extracted lifetime components and amplitudes together with the integrated $\mathrm{E}_{11}$ * to $\mathrm{E}_{11} \mathrm{PL}$ intensities for each defect type. We find that the amplitude-averaged $\mathrm{E}_{11} *$ lifetime $\left(\tau_{\text {amp-av }}\right)$ of PTM defects increases by a factor of 2.4 upon radical decomposition, which is in excellent agreement with the 2.5 -fold enhancement of the $\mathrm{E}_{11} * / \mathrm{E}_{11}$ PL intensity ratio. 
Table 1. PL Lifetime Components (Long: $\tau_{\mathrm{l}}$, Short: $\tau_{\mathrm{s}}$ ) with Corresponding Amplitudes $\left(A_{1}\right.$ and $\left.A_{\mathrm{s}}\right)$, AmplitudeAveraged Lifetimes $\left(\tau_{\text {amp-av }}\right)$, and Integrated Defect-to- $E_{11}$ Intensity Ratio $\operatorname{PL}\left(\mathrm{E}_{11} * / \mathrm{E}_{11}\right)$

\begin{tabular}{lcccccc}
\multicolumn{1}{c}{ defect } & $\begin{array}{c}\mathrm{A}_{1} \\
(\%)\end{array}$ & $\begin{array}{c}\tau_{1} \\
(\mathrm{ps})\end{array}$ & $\begin{array}{c}\mathrm{A}_{\mathrm{s}} \\
(\%)\end{array}$ & $\begin{array}{c}\tau_{\mathrm{s}} \\
(\mathrm{ps})\end{array}$ & $\begin{array}{c}\tau_{\text {amp-av }} \\
(\mathrm{ps})\end{array}$ & $\begin{array}{c}\mathrm{PL} \\
\left(\mathrm{E}_{11} * / \mathrm{E}_{11}\right)\end{array}$ \\
PTM & 16 & 162 & 84 & 30 & 51 & 2.6 \\
PTM irrad. & 30 & 245 & 70 & 68 & 121 & 6.5 \\
PTMH & 29 & 239 & 71 & 81 & 127 & 0.6 \\
ArBr & 31 & 275 & 69 & 95 & 150 & 3.7 \\
\hline
\end{tabular}

PL Quenching Mechanism. In the following, we discuss the possible underlying mechanisms of quenching of the defect-localized exciton by the radical substituent on the aryl defect. Three mechanisms must be considered: resonant excitation energy transfer, photoinduced electron transfer, and enhanced intersystem crossing. First, the possibility of energy transfer from the $\mathrm{sp}^{3}$ defect state to the PTM radical can be ruled out based on the much smaller optical gap of the defect compared to the PTM and negligible spectral overlap between the two partners, as shown in Supporting Information Figure S7.

Second, in order to assess the potential for photoinduced electron transfer (PET), the redox potentials of all involved species and their optical transition energies must be considered (see Table 2). From these data, the Gibbs free energy for PET

Table 2. Redox Potentials $\left(E_{\text {ox }}, E_{\text {red }}\right)$, Optical Transition Energies $\left(\Delta G_{00}\right)$, and Gibbs Free Energies for Photoinduced Electron Transfer $\left(\Delta G_{\mathrm{PET}}\right)$

\begin{tabular}{lcccc}
\multicolumn{1}{c}{ species } & $E_{\text {ox }}(\mathrm{V})^{a}$ & $E_{\text {red }}(\mathrm{V})^{a}$ & $\Delta G_{00}(\mathrm{eV})^{c}$ & $\Delta G_{\text {PET }}(\mathrm{eV})^{d}$ \\
(6,5) pristine & 0.615 & -0.420 & 1.24 & $-2.17^{e}$ \\
$(6,5) \mathrm{sp}^{3}$ defect $^{b}$ & 0.584 & -0.398 & 1.06 & $-1.95^{f}$ \\
PTM & 1.61 & -0.19 & &
\end{tabular}

${ }^{a}$ Potentials from Shiraishi et al. ${ }^{40}$ and Souto et al. ${ }^{41}$ measured versus a $\mathrm{Ag} / \mathrm{AgCl}$ reference electrode. ${ }^{b}$ Data from Shiraishi et al. ${ }^{40}$ for $\mathrm{ArBr}$ defects were used as an approximation to PTM-substituted aryl defects because of their near-identical optical trap depths. ${ }^{c}$ Energy of the $\mathrm{E}_{11}$ and $\mathrm{E}_{11} * \mathrm{PL}$ transition, respectively. ${ }^{d}$ Including an electrostatic work term of $-0.5 \mathrm{eV}$. ${ }^{e}$ For the reaction: SWCNT + $\mathrm{PTM} \rightarrow \mathrm{SWCNT}^{+}+\mathrm{PTM}^{-} .{ }^{f}$ For the reaction: defect $+\mathrm{PTM} \rightarrow$ defect $^{+}+\mathrm{PTM}^{-}$. from pristine or $\mathrm{sp}^{3}$-functionalized regions of the SWCNT (donor D) to the PTM (acceptor A) was evaluated according to ${ }^{37}$

$$
\Delta G_{\mathrm{PET}}=e E_{\mathrm{red}}\left(D^{+} / D\right)-e E_{\mathrm{red}}\left(A / A^{-}\right)-\Delta G_{00}-\frac{e^{2}}{4 \pi \varepsilon_{r} \varepsilon_{0} d}
$$

where $E_{\text {red }}$ denotes the reduction potential, $\Delta G_{00}$ is the optical transition energy, and the electrostatic work term amounts to $0.5 \mathrm{eV}$ for the relative permittivity of toluene $\left(\varepsilon_{r}=2.38\right)$ and an ion pair separation $d \approx 1.2 \mathrm{~nm}$. After PET to the functional group, the electron is back-transferred to the ground state of the defect/SWCNT, thereby resulting in nonradiative recombination. As indicated by a large negative $\Delta G_{\mathrm{PET}}$ in Table 2, PET is thermodynamically highly favorable ${ }^{38}$ and could potentially quench both $\mathrm{E}_{11}$ and $\mathrm{E}_{11} *$ emission. However, since the rate of electron transfer reactions decreases exponentially on a length scale of $\sim 0.1 \mathrm{~nm},{ }^{39}$ the quenching of mobile $\mathrm{E}_{11}$ excitons can be excluded due to their long average distance to the radical, in agreement with negligible $\mathrm{E}_{11}$ brightening in Figure 4a. The defect-localized exciton, on the other hand, is sufficiently close to the PTM to be quenched via PET, even though the separation of $\sim 1 \mathrm{~nm}$ is likely to limit the rate of this process. For the sake of completeness, it is worth noting that the redox potentials in Table 2 permit ground state charge transfer from the SWCNT/defect to the PTM as well, but such a permanent ion pair formation at the defect site is irreconcilable with the observation of the EPR signature from the PTM defect and the PL lifetime shortening, which indicates dynamic rather than static quenching.

As another potential mechanism, covalently bound radicals have been shown to substantially speed up intersystem crossing in organic fluorophores. ${ }^{42-45}$ This effect can be modeled by treating the electronic exchange interaction as a perturbation that promotes spin flips of the unpaired electron on the radical and the photoexcited electron on the chromophore. ${ }^{46}$ The intersystem crossing rate thus depends sensitively on the degree of wave function overlap between the singly occupied molecular orbital (SOMO) on the radical and the singlet exciton. It can reach time scales of $\sim 1-100$ ps for not too distant radicals. ${ }^{42-45}$ Hence, this mechanism is similarly shortranged to PET and could affect defect-localized excitons close to the PTM radical. As discussed below, such radical-enhanced intersystem crossing (EISC) would allow triplet states to a)

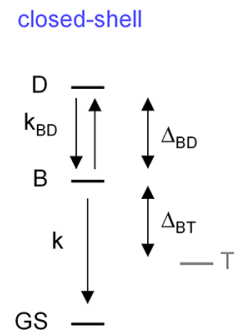

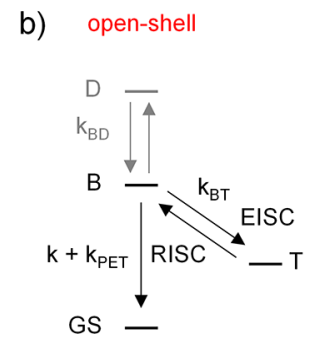

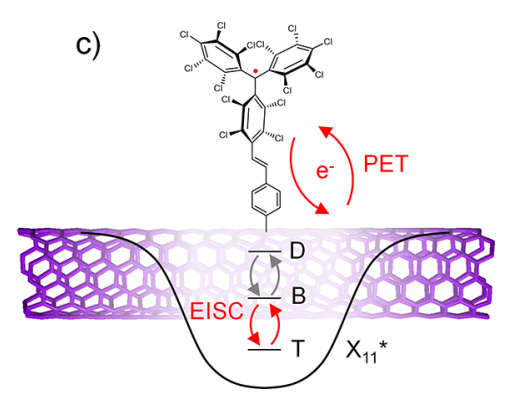

Figure 5. Model of exciton dynamics in SWCNTs with $\mathrm{sp}^{3}$ defects with (a) closed-shell or (b) open-shell functional groups. The energy level diagrams include the ground state (GS), the defect-localized bright (B) and dark (D) singlet states, and a triplet (T) state with energy gaps $\Delta_{\mathrm{BD}}$ and $\Delta_{\mathrm{BT}}$. The relevant rate constants are indicated as follows: $k$, radiative and nonradiative recombination; $k_{\mathrm{PET}}$, photoinduced electron transfer (PET); $k_{\mathrm{BD}}$, equilibration between $\mathrm{B}$ and $\mathrm{D}$ state population; $k_{\mathrm{BT}}$, equilibration between $\mathrm{B}$ and $\mathrm{T}$ state population via radicalenhanced intersystem crossing (EISC) and thermally activated reverse intersystem crossing (RISC). Note that $k_{\mathrm{BD}}$ and $k_{\mathrm{BT}}$ are not the transition rates between states, but represent the relaxation rates at which the involved populations approach their equilibrium value. The rate of this relaxation process depends on the elementary transition rates. Gray color marks states with small population or rates that are not competitive. (c) Schematic illustration of PET and EISC as the PL quenching processes in open-shell PTM defects. 
a)

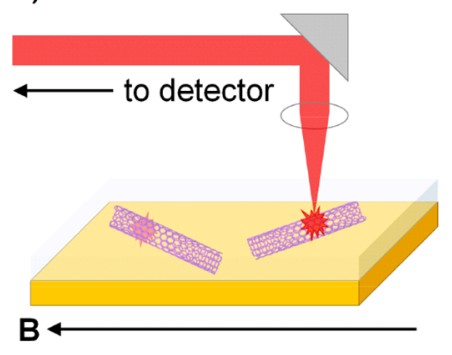

b)

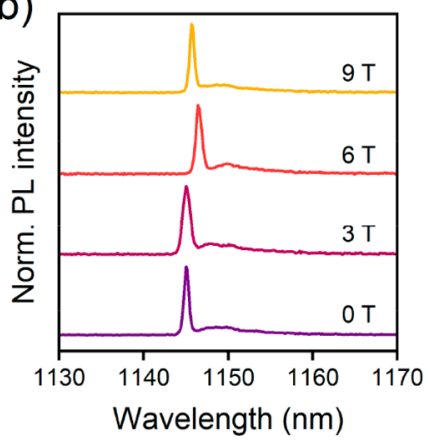

c)

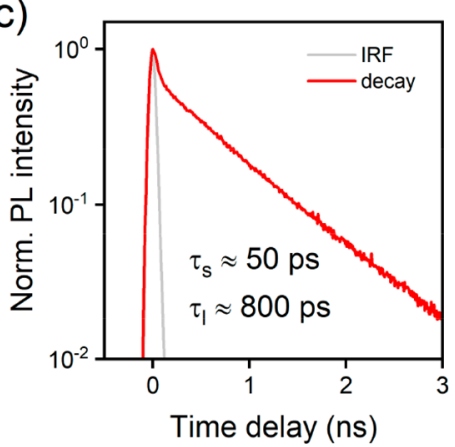

Figure 6. (a) Schematic of confocal magneto-PL measurement in Voigt geometry. (b) Magnetic field-dependent PL spectra of a single PTMfunctionalized $(6,5)$ nanotube (angle between tube axis and magnetic field: $60^{\circ}$ ). Note that minor shifts of the emission wavelength are due to spectral jitter and do not correlate with the applied field. (c) Low-temperature PL decay of the defect signal shown in (b) at zero field. All measurements were performed at $4 \mathrm{~K}$.

participate in the fast population redistribution process between bright and dark defect states following exciton trapping. ${ }^{33,34,47}$ In this case, the radical-induced PL quenching may be explained by exciton population transfer from the bright singlet to a dark triplet state.

Both the PET and the EISC mechanisms are likely to occur for PTM defects based on thermodynamic considerations and previous work on structurally similar molecular systems. To further corroborate or exclude the involvement of either quenching mechanism, we now discuss the $\mathrm{E}_{11} * \mathrm{PL}$ decay in more detail, as it is rich in information and data are available for both radical-functionalized and reference defects (Table 1). Hartmann et l. $^{34}$ and He et al. ${ }^{33}$ developed a model for the biexponential decay of excitons localized at defects with closedshell substituents at room temperature. Since the $\mathrm{E}_{11} * \mathrm{PL}$ decay of radical-functionalized defects remains biexponential with well-separated long and short time scales, we assume that the dynamics in such defects are still similar to the existing model but should be modified by introducing PET or EISC. With these assumptions, the observed radical-induced changes in the long and short decay components can only be explained by a combination of PET and reversible EISC to a triplet state that is energetically below the bright state, as discussed in detail in the Supporting Information.

Figure 5 illustrates our proposed model for defects with open-shell substituents and compares it with the model that is generally applied for defects with closed-shell substituents. $^{33,34,47}$ The involved states are the defect-localized bright singlet exciton (B) and parity-forbidden dark singlet exciton (D), as well as a triplet exciton ( $\mathrm{T})$, which could be either mobile or defect-localized. These states are assumed to reversibly exchange population at room temperature as the energy gaps between them are either smaller $\left(\Delta_{\mathrm{BD}} \approx 9 \mathrm{meV}\right.$ as found by Kim et al. ${ }^{47}$ ) or not much larger than $25 \mathrm{meV}$ (an upper boundary of $\Delta_{\mathrm{BT}} \lesssim 38 \mathrm{meV}$ will be estimated below). In general, the fast decay component is attributed to the redistribution of exciton population among these bright and dark states, which is followed by the slow recombination of trapped excitons via radiative and nonradiative channels. More specifically, as intersystem crossing is slow in the absence of the radical (Figure 5a); only the $\mathrm{D}$ state accepts population from the $\mathrm{B}$ state with the redistribution rate $k_{\mathrm{BD}}$. Subsequently, trapped excitons recombine via multiphonon decay and other pathways with the cumulative rate $k=\tau_{1}^{-1}$, while the ratio of $\mathrm{B}$ and D state populations retains its equilibrium value. Since recombination can occur at any time after the trapping event, the short decay component is given by $\tau_{\mathrm{s}}^{-1}=k_{\mathrm{BD}}+\tau_{1}^{-1}$. In contrast to that, we propose that radical functionalization (Figure $5 \mathrm{~b}$ ) enables fast EISC and thermally activated reverse intersystem crossing (RISC) between the B state and an energetically low-lying $\mathrm{T}$ state. As a result, this $\mathrm{T}$ state dominates the population redistribution process among bright and dark states (for details see Supporting Information). Simultaneously, PET is responsible for enhanced nonradiative recombination, resulting in $\tau_{1}^{-1}=k+k_{\mathrm{PET}}$ and $\tau_{\mathrm{s}}^{-1}=k_{\mathrm{BT}}+\tau_{1}^{-1}$ for radical-substituted defects.

A quantitative analysis of the data in Table 1 further supports this picture. Assuming that PET is responsible for changes in the decay rates $\tau_{1}^{-1}$, a PET rate of $\tau_{1}^{-1}(\mathrm{PTM})-$ $\tau_{1}^{-1}(\mathrm{PTM}$ irrad. $)=(500 \mathrm{ps})^{-1}$ is extracted. This moderately fast electron transfer rate is rationalized by the trade-off between the large thermodynamic driving force for the process $\left(\Delta G_{\mathrm{PET}}=-1.95 \mathrm{eV}\right)$ and the relatively long distance $(\sim 1.2$ $\mathrm{nm}$ ) between the SWCNT surface and the radical center. Further, the term $\left(\tau_{s}^{-1}-\tau_{1}^{-1}\right)$ yields the population redistribution rates $k_{\mathrm{BD}}=(91 \mathrm{ps})^{-1}$ and $k_{\mathrm{BT}}=(37 \mathrm{ps})^{-1}$ for the closed- and open-shell case, respectively. Note that the calculated $k_{\mathrm{BT}}$ is in good agreement with EISC rates ${ }^{43,45}$ for chromophore-radical distances of $1 \mathrm{~nm}$ as present in PTM defects. As pointed out by $\mathrm{He}$ et al., ${ }^{33}$ the normalized amplitude of the long decay component may be used as a measure of bright state population in equilibrium with respect to its initial population immediately after trapping (Supporting Information). For radical-functionalized defects $A_{1}$ is $16 \%$, whereas $A_{1}$ is about $30 \%$ across all reference defects, in good agreement with previous reports. ${ }^{33}$ This reduction of $A_{1}$ further supports EISC-mediated population transfer from the B to the $\mathrm{T}$ state as a contribution to $\mathrm{E}_{11} * \mathrm{PL}$ quenching. Moreover, because the equilibrium populations of the $\mathrm{B}, \mathrm{D}$, and $\mathrm{T}$ states should simply reflect the Boltzmann distribution, we can use the information that $A_{1}=16 \%$ together with $\Delta_{\mathrm{BD}} \approx 9 \mathrm{meV}$ for $\mathrm{E}_{11} *$ defects in $(6,5)$ SWCNTs ${ }^{47}$ to obtain a rough estimate of $\Delta_{\mathrm{BT}} \approx 38 \mathrm{meV}$. So far, we assumed a single triplet state to be involved, although several triplet states are predicted ${ }^{48}$ within a few $10 \mathrm{meV}$ of the bright $\mathrm{E}_{11}$ * singlet. Including a second triplet state will further reduce $\Delta_{\mathrm{BT}}$, so $38 \mathrm{meV}$ should be regarded as an upper boundary, which justifies the earlier assumption of RISC at room temperature. Finally, we note that within this model about $\sim 1 / 3$ of the defect PL intensity drop results from PET, whereas $\sim 2 / 3$ are a consequence of EISC (Supporting Information). It is important to emphasize that our description of the decay dynamics of radical-functionalized 
defects and the extracted physical parameters only apply as long as the underlying model for closed-shell defects ${ }^{33,34}$ is valid. For example, if the biexponential decay instead resulted from shelving into dark states, similar to the case of $E_{11}$ excitons, ${ }^{49,50}$ other quenching mechanisms might match the data more closely.

As long lifetimes $(>1 \mu \mathrm{s})$ are a hallmark of triplet states in organic materials, we also looked for delayed fluorescence at room temperature. ${ }^{51,52}$ To this end, the PL decay of PTMfunctionalized SWCNTs was monitored at the $\mathrm{E}_{11}$ or $\mathrm{E}_{11}$ * peak emission wavelength by TCSPC over a time window of $0.3 \mu \mathrm{s}$. However, in neither case was a component with $\mu$ s lifetime detectable. While it is possible that the delayed fluorescence signal is simply too weak to be detected in our setup, it is important to keep in mind that RISC (from the T to the B state) is enhanced by the same factor as the forward process. Thus, their efficient coupling via EISC and RISC should lead to simultaneous depopulation of the $\mathrm{B}$ and the $\mathrm{T}$ state on the time scale of $\tau_{1}(\sim 100 \mathrm{ps})$. Hence, the effective triplet state lifetime is strongly reduced and delayed fluorescence is no longer expected.

Magneto-photoluminescence Spectroscopy. Given the clear evidence for interaction of the radical with the trapped exciton, we explored how the paramagnetic PTM substituent influences the PL of $\mathrm{sp}^{3}$ defects in magnetic fields. Low-temperature magneto-PL experiments were performed on individual, PTM-functionalized $(6,5)$ SWCNTs embedded in a polystyrene layer (see Supporting Information Figure S8 for a scanning confocal PL image). As illustrated in Figure 6a, the sample was mounted such that PL could be collected while applying an in-plane magnetic field (Voigt geometry). The random nanotube orientation results in a tube-specific angle between the tube axis and the magnetic field. In total, eight nanotubes were investigated that displayed single-line or wellseparated multiline defect PL between 1120 and $1180 \mathrm{~nm}$ $(1.05-1.11 \mathrm{eV})$. Note that the sidebands that are red-shifted by $2-3 \mathrm{meV}$ from the zero-phonon line are typical of the cryogenic PL spectra of polymer-wrapped SWCNTs. ${ }^{53,54}$

$\mathrm{Up}$ to magnetic fields of $9 \mathrm{~T}$, none of these defect signals displayed a significant shift or splitting within the resolution limit of the spectrometer $(\sim 300 \mu \mathrm{eV})$ and the characteristic spectral jitter $(\sim 2 \mathrm{meV})$ as exemplified in Figure $6 \mathrm{~b}$ and more generally shown in Supporting Information Figure S9. This result is slightly surprising, because Kim et al. recently observed magnetic field-induced fine structure in the spectra of $\mathrm{sp}^{3}$ defects. ${ }^{48}$ Yet, peak splitting only occurred in a minority of nanotubes, and the authors hypothesized that this was related to the requirement of nearly perfect energy level alignment between the bright $\mathrm{E}_{11} *$ singlet and the band-edge triplet exciton. Within our limited statistics we cannot rule out that a fraction of nanotubes in our sample would show a similar effect, but based on the above estimation that $\Delta_{\mathrm{BT}} \lesssim 38 \mathrm{meV}$, it is likely that there is indeed no triplet state within just a few $\mathrm{meV}$ of the bright state in our PTM defects, which would explain the absence of a peak splitting. It is also worth noting that the triplet state, which is populated via EISC from an energetically close singlet state, usually does not show enhanced phosphorescence in magnetic fields, because the EISC rate also depends on the energy gap between the involved states ${ }^{46}$ and the gap between the excited triplet and the singlet ground state is too large to result in significant triplet brightening. ${ }^{42}$ Beyond that, the spin Zeeman effect that acts on the radical and splits its SOMO level is insufficient to induce a change of the defect $\mathrm{PL}$, as the unpaired electron on the PTM is merely a spectator to the recombination of the defect-localized exciton with zero spin. Conceptually, the situation is analogous to that of defect-trapped trions in SWCNTs. ${ }^{55}$ In both cases, the spin state does not change upon optical transition from the excited to the ground state, thus resulting in a vanishing spin Zeeman effect.

To gain further insights into the energetic position of the triplet state, we measured the PL decay of an individual PTM defect at $4 \mathrm{~K}$ (Figure 6c). Interestingly, we observed a biexponential decay characterized by an unusually ${ }^{47,56}$ fast component $\left(\tau_{\mathrm{s}} \approx 50 \mathrm{ps}\right)$ with large amplitude $\left(A_{\mathrm{s}} \approx 60 \%\right)$ and a slow component $\left(\tau_{1} \approx 800 \mathrm{ps}\right)$ typical for trapped exciton recombination at low temperature. Such a dominant fast process has never been reported for aryl defects with closedshell substituents at low temperature. Note that multiexciton effects can be excluded as the origin of the fast decay component, because the pump fluence $\left(\sim 2 \times 10^{14}\right.$ photons $\mathrm{cm}^{-2}$ pulse $^{-1}$ ) was kept within the regime of linear PL response. $^{57}$ Since PET is too slow in our system while the observed $\tau_{\mathrm{s}}$ matches the room-temperature EISC rate well, we assign the fast decay component to EISC and conclude that the participating triplet state is energetically below the bright state. However, since RISC is prohibited at $4 \mathrm{~K}$, the origin of $\tau_{1}$ is not obvious. We speculate that the surrounding insulating polymer matrix and cryogenic temperature could facilitate the trapping of charges on the PTM group, e.g., after a PET event. This leads to electrochemical reduction of the radical to the closedshell anion, in which EISC is switched off. In this case, $\tau_{\text {s }}$ corresponds to the decay time when the defect is in its native radical state, whereas $\tau_{1}$ represents the slower decay when the defect is trapped in its spin-paired anion state without EISC. Nevertheless, further work will be required to fully understand the PL dynamics of radical-functionalized defects in SWCNTs at cryogenic temperatures.

\section{CONCLUSION}

In summary, we have demonstrated the functionalization of monochiral semiconducting SWCNTs with stable organic radicals via simple diazonium chemistry. The presence of the radical leads to partial quenching of defect PL, which is likely due to a combination of a photoinduced electron transfer process and population transfer to triplet states enabled by radical-enhanced intersystem crossing. Consequently, radicalfunctionalized $\mathrm{sp}^{3}$ defects are an interesting platform for future studies of the elusive triplet exciton manifold in carbon nanotubes employing spin-sensitive methods such as optically detected magnetic resonance. ${ }^{51,58}$ Moreover, as pulsed EPR techniques can probe the distances between spin labels on the length scale of a few nanometers, ${ }^{59}$ such defects could be a model system for investigating clustering, which is suspected to play an important part in both synthetic ${ }^{60,61}$ and optical ${ }^{56}$ features of luminescent defects. Beyond that, the encapsulation of such radical-tailored SWCNTs in biocompatible surfactants ${ }^{62}$ might even enable their use as metal-free contrast agents for magnetic resonance imaging ${ }^{63,64}$ complementary to in vivo near-infrared fluorescence imaging. ${ }^{2}$ Finally, our functionalization approach should be easily transferable to metallic SWCNTs, which show even higher reactivity toward diazonium reagents ${ }^{65}$ and are commonly used in spin valves. In order to maximize the coupling between the radical and the itinerant electrons in such a system, the covalent linker should 
be as short as possible to place the radical close to the conducting channel.

\section{METHODS}

Selective Dispersion of $(6,5)$ SWCNTs. As described previously, ${ }^{24}(6,5)$ SWCNTs were extracted from CoMoCAT raw material (Chasm Advanced Materials, SG65i-L63, $0.38 \mathrm{~g} \mathrm{~L}^{-1}$ ) by selective wrapping with poly-[(9,9-dioctylfluorenyl-2,7-diyl)-alt$\left(6,6^{\prime}\right)-\left(2,2^{\prime}\right.$-bipyridine)] (PFO-BPy, American Dye Source, $\mathrm{M}_{\mathrm{w}}=$ $40 \mathrm{~kg} \mathrm{~mol}^{-1}, 0.5 \mathrm{~g} \mathrm{~L}^{-1}$ ) under shear force mixing (Silverson L2/Air, $10230 \mathrm{rpm}, 72 \mathrm{~h}$ ) in toluene. Aggregates and impurities were removed by two centrifugation steps $(60000 \mathrm{~g}$ for $45 \mathrm{~min}$ each) and subsequent filtration (poly(tetrafluoroethylene) (PTFE) syringe filter, $5 \mu \mathrm{m}$ pore size).

SWCNT Functionalization. $(6,5)$ SWCNTs were reacted with a series of diazonium compounds to introduce $\mathrm{sp}^{3}$ aryl defects with either radical (PTM) or closed-shell substituents (PTMH, Br). Since the employed diazonium salts displayed different solubility characteristics, the functionalization procedure was adapted to this. The reactions between $(6,5)$ SWCNTs and the tailored diazonium salts PTM-Dz and PTMH-Dz (see the Supporting Information for the synthesis $^{26}$ and characterization) were performed in THF, which was freshly distilled to remove water and any peroxide contaminations. First, the PFO-BPy-wrapped $(6,5)$ SWCNTs were transferred to THF by passing the toluene dispersion through a PTFE membrane filter (Merck Millipore, JVWP, $0.1 \mu \mathrm{m}$ pore size) to collect the SWCNTs, which were subsequently redispersed in a small volume of THF by bath sonication. To this dispersion was added a solution of the diazonium salt (PTM-Dz or PTMH-Dz) in THF such that the $(6,5)$ SWCNT concentration in the reaction mixture was $0.72 \mathrm{mg} \mathrm{L}^{-1}$ (optical density of $0.4 \mathrm{~cm}^{-1}$ at the $\mathrm{E}_{11}$ transition) and the diazonium salt concentration $0.15 \mathrm{mmol} \mathrm{L}^{-1}$. In contrast to that, the reaction with the commercial 4-bromobenzenediazonium tetrafluoroborate (Br-Dz, Sigma-Aldrich) was carried out according to our previously reported procedure ${ }^{27}$ using 18 -crown- 6 as a phase transfer agent in an $80: 20 \mathrm{vol} \%$ toluene/acetonitrile mixture, but without the addition of potassium acetate. The $(6,5)$ SWCNT concentration was adjusted to $0.72 \mathrm{mg} \mathrm{L}^{-1}$ and the $\mathrm{Br}-\mathrm{Dz}$ concentration to $0.37 \mathrm{mmol} \mathrm{L}^{-1}$ for moderate- or $1.85 \mathrm{mmol} \mathrm{L}^{-1}$ for high-density functionalization. All reactions proceeded in air at room temperature under dark conditions. After $16 \mathrm{~h}$, the functionalized SWCNTs were collected by vacuum filtration through a PTFE membrane filter (Merck Millipore, JVWP, $0.1 \mu \mathrm{m}$ pore size) and washed with either THF $(3 \times$ $7 \mathrm{~mL})$ or acetonitrile $(10 \mathrm{~mL})$ followed by toluene $(5 \mathrm{~mL})$, depending on the reaction medium. For analysis, the functionalized SWCNTs were redispersed in a small volume of toluene by bath sonication for $30 \mathrm{~min}$.

EPR Spectroscopy. EPR spectra were recorded at room temperature on a Bruker ESP $300 \mathrm{E}$ spectrometer provided with a $\mathrm{T} 102$ rectangular cavity that works with an $\mathrm{X}$ band $(9.5 \mathrm{GHz})$. The signal-to-noise ratio of spectra was increased by accumulation of scans using the F/Flock accessory to guarantee large field reproducibility. Precautions to avoid undesirable spectral distortion and line broadenings, such as those arising from microwave power saturation and magnetic field overmodulation, were also taken into account to improve sensitivity.

Room-Temperature PL Characterization. PL spectra and TCSPC were measured for SWCNT dispersions using a home-built setup. The samples were excited by the spectrally filtered output of a picosecond-pulsed supercontinuum laser source (Fianium WhiteLase SC400) tuned to $700 \mathrm{~nm}$ to avoid any photochemical reactions of PTM-functionalized SWCNTs. Scattered laser light was blocked by appropriate long-pass filters. The sample emission was dispersed by a grating spectrograph (Acton SpectraPro SP2358, 150 lines $\mathrm{mm}^{-1}$ ) and detected by a liquid nitrogen cooled InGaAs line camera (Princeton Instruments OMA V:1024-1.7 LN). For TCSPC, the spectrally selected output of the spectrograph was focused onto a gated InGaAs/InP avalanche photodiode (Micro Photon Devices). Histograms of photon arrival times were created using a counting module (PicoHarp 300, PicoQuant) and reconvolution-fitted using the SymPhoTime 64 software. The instrument response function (IRF) was determined by the fast, instrument-limited decay of the $\mathrm{E}_{11}$ emission from $(6,5)$ SWCNTs.

Low-Temperature Magneto-spectroscopy. For PL microscopy on the single-defect level, a low concentration of functionalized $(6,5)$ SWCNTs (optical density of $0.005 \mathrm{~cm}^{-1}$ at the $\mathrm{E}_{11}$ transition) was blended into a solution of polystyrene $\left(20 \mathrm{~g} \mathrm{~L}^{-1}\right)$ in toluene and spin-coated at $2000 \mathrm{rpm}$ onto glass slides coated with $150 \mathrm{~nm}$ of gold. The sample was loaded into a home-built confocal microscope setup inside a closed-cycle magneto-cryostat (attoDRY1000, attocube systems AG) with a base temperature of $3.2 \mathrm{~K}$ and magnetic fields of up to $9 \mathrm{~T}$. A customized holder was used to mount the sample in Voigt geometry with the magnetic field parallel and light propagation orthogonal to the sample surface. A wavelength-tunable Ti:sapphire laser (Coherent Mira) served as the excitation source and was tuned to $995 \mathrm{~nm}$ (continuous wave, $6 \mu \mathrm{W}$ ) to be in resonance with the $\mathrm{E}_{11}$ transition of $(6,5)$ SWCNTs. The PL from individual nanotubes was collected by an aspheric lens (Thorlabs 354330-B, NA = 0.68), dispersed by a grating spectrograph (Acton SpectraPro SP2500, 300 lines $\mathrm{mm}^{-1}$ ) and recorded by a liquid nitrogen cooled InGaAs line camera (Princeton Instruments OMA V:1024-1.7 LN). For TCSPC, nanotubes were excited in pulsed mode $\left(\sim 2 \times 10^{14}\right.$ photons $\mathrm{cm}^{-2}$ pulse $\left.^{-1}\right)$ and the PL was directed to a superconducting single-photon detector (TCOPRS-CCR-SW-85, Scontel). The IRF was measured on the attenuated laser signal.

\section{ASSOCIATED CONTENT}

\section{SI Supporting Information}

The Supporting Information is available free of charge at https://pubs.acs.org/doi/10.1021/acsnano.0c10341.

Optical trap depth of PTM vs PTMH defects, highly functionalized closed-shell reference, fine structure of the EPR signal, broadening of the EPR signal, proposed reaction scheme for open-shell to closed-shell conversion, normalized spectra for irradiation experiment, exclusion of energy transfer due to negligible spectral overlap, model for PL decay dynamics of $\mathrm{sp}^{3}$ defects with open-shell substituents, scanning confocal PL map of single nanotubes, magneto-PL spectra of single nanotubes, synthesis and characterization of diazonium compounds including raw spectral data for PTMH-Dz (PDF)

\section{AUTHOR INFORMATION}

\section{Corresponding Author}

Jana Zaumseil - Institute for Physical Chemistry, Universität Heidelberg, 69120 Heidelberg, Germany; Centre for Advanced Materials, Universität Heidelberg, 69120 Heidelberg, Germany; ○ orcid.org/0000-0002-2048-217X; Email: zaumseil@uni-heidelberg.de

\section{Authors}

Felix J. Berger - Institute for Physical Chemistry, Universität Heidelberg, 69120 Heidelberg, Germany; Centre for Advanced Materials, Universität Heidelberg, 69120 Heidelberg, Germany

J. Alejandro de Sousa - Institut de Ciència de Materials de Barcelona (ICMAB-CSIC), 08193 Bellaterra, Spain; Laboratorio de Electroquímica, Departamento de Química, Facultad de Ciencias, Universidad de los Andes, 5101 Mérida, Venezuela; 이이.org/0000-0002-7948-8162

Shen Zhao - Faculty of Physics, Munich Quantum Center and Center for NanoScience (CeNS), Ludwig-MaximiliansUniversität München, 80539 München, Germany; Munich 
Center for Quantum Science and Technology (MCQST), 80799 München, Germany

Nicolas F. Zorn - Institute for Physical Chemistry, Universität Heidelberg, 69120 Heidelberg, Germany; Centre for Advanced Materials, Universität Heidelberg, 69120 Heidelberg, Germany

Abdurrahman Ali El Yumin - Institute for Physical Chemistry, Universität Heidelberg, 69120 Heidelberg, Germany; Centre for Advanced Materials, Universität Heidelberg, 69120 Heidelberg, Germany

Aleix Quintana García - Institut de Ciència de Materials de Barcelona (ICMAB-CSIC), 08193 Bellaterra, Spain

Simon Settele - Institute for Physical Chemistry, Universität Heidelberg, 69120 Heidelberg, Germany

Alexander Högele - Faculty of Physics, Munich Quantum Center and Center for NanoScience (CeNS), LudwigMaximilians-Universität München, 80539 München, Germany; Munich Center for Quantum Science and Technology (MCQST), 80799 München, Germany; (1) orcid.org/0000-0002-0178-9117

Núria Crivillers - Institut de Ciència de Materials de Barcelona (ICMAB-CSIC), 08193 Bellaterra, Spain; (1) orcid.org/0000-0001-6538-2482

Complete contact information is available at:

https://pubs.acs.org/10.1021/acsnano.0c10341

\section{Notes}

The authors declare no competing financial interest.

\section{ACKNOWLEDGMENTS}

The authors thank Dr. Vega Lloveras for performing electron paramagnetic resonance measurements. This project has received funding from the European Research Council (ERC) under the European Union's Horizon 2020 research and innovation programme (grant agreement no. 817494 "TRIFECTs"). S.Z. acknowledges funding from the Alexander von Humboldt Foundation, and A.H. from the European Research Council (ERC) under the grant agreement no. 772195 and the Deutsche Forschungsgemeinschaft (DFG, German Research Foundation) under Germany's Excellence Strategy EXC-2111-390814868. This work was also supported by the MICIU of Spain (PID2019-111682RB-I00), the Generalitat de Catalunya (2017-SGR-918), and the Severo Ochoa FUNFUTURE (CEX2019-000917-S). J.A.d.S. is enrolled in the Materials Science Ph.D. program of UAB. J.A.d.S. thanks the FPI fellowship.

\section{REFERENCES}

(1) Luo, Y.; He, X.; Kim, Y.; Blackburn, J. L.; Doorn, S. K.; Htoon, H.; Strauf, S. Carbon Nanotube Color Centers in Plasmonic Nanocavities: A Path to Photon Indistinguishability at Telecom Bands. Nano Lett. 2019, 19, 9037-9044.

(2) Mandal, A. K.; Wu, X.; Ferreira, J. S.; Kim, M.; Powell, L. R.; Kwon, H.; Groc, L.; Wang, Y.; Cognet, L. Fluorescent $\mathrm{sp}^{3}$ DefectTailored Carbon Nanotubes Enable NIR-II Single Particle Imaging in Live Brain Slices at Ultra-Low Excitation Doses. Sci. Rep. 2020, 10, 5286.

(3) Lee, Y.; Trocchia, S. M.; Warren, S. B.; Young, E. F.; Vernick, S.; Shepard, K. L. Electrically Controllable Single-Point Covalent Functionalization of Spin-Cast Carbon-Nanotube Field-Effect Transistor Arrays. ACS Nano 2018, 12, 9922-9930.

(4) Durkop, T.; Getty, S. A.; Cobas, E.; Fuhrer, M. S. Extraordinary Mobility in Semiconducting Carbon Nanotubes. Nano Lett. 2004, 4, 35-39.
(5) Hertel, T.; Himmelein, S.; Ackermann, T.; Stich, D.; Crochet, J. Diffusion Limited Photoluminescence Quantum Yields in 1-D Semiconductors: Single-Wall Carbon Nanotubes. ACS Nano 2010, 4, 7161-7168.

(6) Brozena, A. H.; Kim, M.; Powell, L. R.; Wang, Y. Controlling the Optical Properties of Carbon Nanotubes with Organic Colour-Centre Quantum Defects. Nat. Rev. Chem. 2019, 3, 375-392.

(7) Shiraki, T.; Miyauchi, Y.; Matsuda, K.; Nakashima, N. Carbon Nanotube Photoluminescence Modulation by Local Chemical and Supramolecular Chemical Functionalization. Acc. Chem. Res. 2020, 53, 1846-1859.

(8) Gifford, B. J.; Kilina, S.; Htoon, H.; Doorn, S. K.; Tretiak, S. Controlling Defect-State Photophysics in Covalently Functionalized Single-Walled Carbon Nanotubes. Acc. Chem. Res. 2020, 53, 17911801.

(9) Ghosh, S.; Bachilo, S. M.; Simonette, R. A.; Beckingham, K. M.; Weisman, R. B. Oxygen Doping Modifies Near-Infrared Band Gaps in Fluorescent Single-Walled Carbon Nanotubes. Science 2010, 330, $1656-1659$.

(10) Piao, Y.; Meany, B.; Powell, L. R.; Valley, N.; Kwon, H.; Schatz, G. C.; Wang, Y. Brightening of Carbon Nanotube Photoluminescence through the Incorporation of $\mathrm{sp}^{3}$ Defects. Nat. Chem. 2013, 5, 840845 .

(11) Kwon, H.; Furmanchuk, A.; Kim, M.; Meany, B.; Guo, Y.; Schatz, G. C.; Wang, Y. Molecularly Tunable Fluorescent Quantum Defects. J. Am. Chem. Soc. 2016, 138, 6878-6885.

(12) He, X.; Hartmann, N. F.; Ma, X.; Kim, Y.; Ihly, R.; Blackburn, J. L.; Gao, W.; Kono, J.; Yomogida, Y.; Hirano, A.; Tanaka, T.; Kataura, H.; Htoon, H.; Doorn, S. K. Tunable Room-Temperature SinglePhoton Emission at Telecom Wavelengths from $\mathrm{sp}^{3}$ Defects in Carbon Nanotubes. Nat. Photonics 2017, 11, 577-582.

(13) Gifford, B. J.; Kilina, S.; Htoon, H.; Doorn, S. K.; Tretiak, S. Exciton Localization and Optical Emission in Aryl-Functionalized Carbon Nanotubes. J. Phys. Chem. C 2018, 122, 1828-1838.

(14) Kwon, H.; Kim, M.; Meany, B.; Piao, Y.; Powell, L. R.; Wang, Y. Optical Probing of Local $\mathrm{pH}$ and Temperature in Complex Fluids with Covalently Functionalized, Semiconducting Carbon Nanotubes. J. Phys. Chem. C 2015, 119, 3733-3739.

(15) Shiraki, T.; Onitsuka, H.; Shiraishi, T.; Nakashima, N. Near Infrared Photoluminescence Modulation of Single-Walled Carbon Nanotubes Based on a Molecular Recognition Approach. Chem. Commun. 2016, 52, 12972-12975.

(16) Onitsuka, H.; Fujigaya, T.; Nakashima, N.; Shiraki, T. Control of the Near Infrared Photoluminescence of Locally Functionalized Single-Walled Carbon Nanotubes via Doping by Azacrown-Ether Modification. Chem. - Eur. J. 2018, 24, 9393-9398.

(17) Mann, F. A.; Herrmann, N.; Opazo, F.; Kruss, S. Quantum Defects as a Toolbox for the Covalent Functionalization of Carbon Nanotubes with Peptides and Proteins. Angew. Chem., Int. Ed. 2020, 59, 17732-17738.

(18) Schnee, M.; Besson, C.; Frielinghaus, R.; Lurz, C.; Kögerler, P.; Schneider, C. M.; Meyer, C. Quantum Transport in Carbon Nanotubes Covalently Functionalized with Magnetic Molecules. Phys. Status Solidi B 2016, 253, 2424-2427.

(19) Ncube, S.; Coleman, C.; Strydom, A.; Flahaut, E.; de Sousa, A.; Bhattacharyya, S. Kondo Effect and Enhanced Magnetic Properties in Gadolinium Functionalized Carbon Nanotube Supramolecular Complex. Sci. Rep. 2018, 8, 8057.

(20) Urdampilleta, M.; Klyatskaya, S.; Cleuziou, J. P.; Ruben, M.; Wernsdorfer, W. Supramolecular Spin Valves. Nat. Mater. 2011, 10, 502-506.

(21) Ji, L.; Shi, J.; Wei, J.; Yu, T.; Huang, W. Air-Stable Organic Radicals: New-Generation Materials for Flexible Electronics? Adv. Mater. 2020, 32, 1908015.

(22) Ratera, I.; Veciana, J. Playing with Organic Radicals as Building Blocks for Functional Molecular Materials. Chem. Soc. Rev. 2012, 41, 303-349.

(23) Ballester, M. Inert Free Radicals (IFR): A Unique Trivalent Carbon Species. Acc. Chem. Res. 1985, 18, 380-387. 
(24) Graf, A.; Zakharko, Y.; Schießl, S. P.; Backes, C.; Pfohl, M.; Flavel, B. S.; Zaumseil, J. Large Scale, Selective Dispersion of Long Single-Walled Carbon Nanotubes with High Photoluminescence Quantum Yield by Shear Force Mixing. Carbon 2016, 105, 593-599.

(25) Seber, G.; Muñoz, J.; Sandoval, S.; Rovira, C.; Tobias, G.; MasTorrent, M.; Crivillers, N. Synergistic Exploitation of the Superoxide Scavenger Properties of Reduced Graphene Oxide and a Trityl Organic Radical for the Impedimetric Sensing of Xanthine. Adv. Mater. Interfaces 2018, 5, 1701072.

(26) Seber, G.; Rudnev, A. V.; Droghetti, A.; Rungger, I.; Veciana, J.; Mas-Torrent, M.; Rovira, C.; Crivillers, N. Covalent Modification of Highly Ordered Pyrolytic Graphite with a Stable Organic Free Radical by Using Diazonium Chemistry. Chem. - Eur. J. 2017, 23, 1415-1421.

(27) Berger, F. J.; Lüttgens, J.; Nowack, T.; Kutsch, T.; Lindenthal, S.; Kistner, L.; Müller, C. C.; Bongartz, L. M.; Lumsargis, V. A.; Zakharko, Y.; Zaumseil, J. Brightening of Long, Polymer-Wrapped Carbon Nanotubes by sp ${ }^{3}$ Functionalization in Organic Solvents. ACS Nano 2019, 13, 9259-9269.

(28) Kim, M.; Adamska, L.; Hartmann, N. F.; Kwon, H.; Liu, J.; Velizhanin, K. A.; Piao, Y.; Powell, L. R.; Meany, B.; Doorn, S. K.; Tretiak, S.; Wang, Y. Fluorescent Carbon Nanotube Defects Manifest Substantial Vibrational Reorganization. J. Phys. Chem. C 2016, 120, 11268-11276.

(29) Zaka, M.; Ito, Y.; Wang, H.; Yan, W.; Robertson, A.; Wu, Y. A.; Rümmeli, M. H.; Staunton, D.; Hashimoto, T.; Morton, J. J. L.; Ardavan, A.; Briggs, G. A. D.; Warner, J. H. Electron Paramagnetic Resonance Investigation of Purified Catalyst-Free Single-Walled Carbon Nanotubes. ACS Nano 2010, 4, 7708-7716.

(30) Weil, J. A.; Bolton, J. R. Electron Paramagnetic Resonance; John Wiley \& Sons: Hoboken, 2007.

(31) Reuel, N. F.; Dupont, A.; Thouvenin, O.; Lamb, D. C.; Strano, M. S. Three-Dimensional Tracking of Carbon Nanotubes within Living Cells. ACS Nano 2012, 6, 5420-5428.

(32) Lohmann, S. H.; Trerayapiwat, K. J.; Niklas, J.; Poluektov, O. G.; Sharifzadeh, S.; Ma, X. sp $^{3}$-Functionalization of Single-Walled Carbon Nanotubes Creates Localized Spins. ACS Nano 2020, 14, 17675-17682.

(33) He, X.; Velizhanin, K. A.; Bullard, G.; Bai, Y.; Olivier, J. H.; Hartmann, N. F.; Gifford, B. J.; Kilina, S.; Tretiak, S.; Htoon, H.; Therien, M. J.; Doorn, S. K. Solvent- and Wavelength-Dependent Photoluminescence Relaxation Dynamics of Carbon Nanotube $\mathrm{sp}^{3}$ Defect States. ACS Nano 2018, 12, 8060-8070.

(34) Hartmann, N. F.; Velizhanin, K. A.; Haroz, E. H.; Kim, M.; Ma, X.; Wang, Y.; Htoon, H.; Doorn, S. K. Photoluminescence Dynamics of Aryl sp ${ }^{3}$ Defect States in Single-Walled Carbon Nanotubes. ACS Nano 2016, 10, 8355-8365.

(35) Fox, M. A.; Gaillard, E.; Chen, C.-C. Photochemistry of Stable Free Radicals: The Photolysis of Perchlorotriphenylmethyl Radicals. J. Am. Chem. Soc. 1987, 109, 7088-7094.

(36) Ballester, M.; Castañer, J.; Riera, J.; Pujadas, J.; Armet, O.; Onrubia, C.; Rio, J. A. Inert Carbon Free Radicals. 5. Perchloro-9Phenylfluorenyl Radical Series. J. Org. Chem. 1984, 49, 770-778.

(37) Weller, A. Photoinduced Electron Transfer in Solution: Exciplex and Radical Ion Pair Formation Free Enthalpies and Their Solvent Dependence. Z. Phys. Chem. 1982, 133, 93-98.

(38) Miller, J. R.; Calcaterra, L. T.; Closs, G. L. Intramolecular LongDistance Electron Transfer in Radical Anions. The Effects of Free Energy and Solvent on the Reaction Rates. J. Am. Chem. Soc. 1984, 106, 3047-3049.

(39) Lewis, F. D.; Wu, T.; Zhang, Y.; Letsinger, R. L.; Greenfield, S. R.; Wasielewski, M. R. Distance-Dependent Electron Transfer in DNA Hairpins. Science 1997, 277, 673-676.

(40) Shiraishi, T.; Shiraki, T.; Nakashima, N. Substituent Effects on the Redox States of Locally Functionalized Single-Walled Carbon Nanotubes Revealed by in Situ Photoluminescence Spectroelectrochemistry. Nanoscale 2017, 9, 16900-16907.

(41) Souto, M.; Cui, H.; Pena-Alvarez, M.; Baonza, V. G.; Jeschke, H. O.; Tomic, M.; Valenti, R.; Blasi, D.; Ratera, I.; Rovira, C.;
Veciana, J. Pressure-Induced Conductivity in a Neutral Nonplanar Spin-Localized Radical. J. Am. Chem. Soc. 2016, 138, 11517-11525.

(42) Wang, Z.; Zhao, J.; Barbon, A.; Toffoletti, A.; Liu, Y.; An, Y.; Xu, L.; Karatay, A.; Yaglioglu, H. G.; Yildiz, E. A.; Hayvali, M. RadicalEnhanced Intersystem Crossing in New Bodipy Derivatives and Application for Efficient Triplet-Triplet Annihilation Upconversion. J. Am. Chem. Soc. 2017, 139, 7831-7842.

(43) Colvin, M. T.; Giacobbe, E. M.; Cohen, B.; Miura, T.; Scott, A. M.; Wasielewski, M. R. Competitive Electron Transfer and Enhanced Intersystem Crossing in Photoexcited Covalent Tempo-Perylene3,4:9,10-Bis(dicarboximide) Dyads: Unusual Spin Polarization Resulting from the Radical-Triplet Interaction. J. Phys. Chem. A 2010, 114, 1741-1748.

(44) Dyar, S. M.; Margulies, E. A.; Horwitz, N. E.; Brown, K. E.; Krzyaniak, M. D.; Wasielewski, M. R. Photogenerated Quartet State Formation in a Compact Ring-Fused Perylene-Nitroxide. J. Phys. Chem. B 2015, 119, 13560-13569.

(45) Giacobbe, E. M.; Mi, Q.; Colvin, M. T.; Cohen, B.; Ramanan, C.; Scott, A. M.; Yeganeh, S.; Marks, T. J.; Ratner, M. A.; Wasielewski, M. R. Ultrafast Intersystem Crossing and Spin Dynamics of Photoexcited Perylene-3,4:9,10-Bis(dicarboximide) Covalently Linked to a Nitroxide Radical at Fixed Distances. J. Am. Chem. Soc. 2009, 131, 3700-3712.

(46) Yeganeh, S.; Wasielewski, M. R.; Ratner, M. A. Enhanced Intersystem Crossing in Three-Spin Systems: A Perturbation Theory Treatment. J. Am. Chem. Soc. 2009, 131, 2268-2273.

(47) Kim, Y.; Velizhanin, K. A.; He, X.; Sarpkaya, I.; Yomogida, Y.; Tanaka, T.; Kataura, H.; Doorn, S. K.; Htoon, H. Photoluminescence Intensity Fluctuations and Temperature-Dependent Decay Dynamics of Individual Carbon Nanotube $\mathrm{sp}^{3}$ Defects. J. Phys. Chem. Lett. 2019, 10, $1423-1430$.

(48) Kim, Y.; Goupalov, S. V.; Weight, B. M.; Gifford, B. J.; He, X.; Saha, A.; Kim, M.; Ao, G.; Wang, Y.; Zheng, M.; Tretiak, S.; Doorn, S. K.; Htoon, H. Hidden Fine Structure of Quantum Defects Revealed by Single Carbon Nanotube Magneto-Photoluminescence. ACS Nano 2020, 14, 3451-3460.

(49) Ishii, A.; Machiya, H.; Kato, Y. K. High Efficiency Dark-toBright Exciton Conversion in Carbon Nanotubes. Phys. Rev. X 2019, 9, 041048.

(50) Gokus, T.; Cognet, L.; Duque, J. G.; Pasquali, M.; Hartschuh, A.; Lounis, B. Mono- and Biexponential Luminescence Decays of Individual Single-Walled Carbon Nanotubes. J. Phys. Chem. C 2010, $114,14025-14028$.

(51) Stich, D.; Späth, F.; Kraus, H.; Sperlich, A.; Dyakonov, V.; Hertel, T. Triplet-Triplet Exciton Dynamics in Single-Walled Carbon Nanotubes. Nat. Photonics 2014, 8, 139-144.

(52) Lin, C. W.; Bachilo, S. M.; Weisman, R. B. Delayed Fluorescence from Carbon Nanotubes through Singlet OxygenSensitized Triplet Excitons. J. Am. Chem. Soc. 2020, 142, 2118921196.

(53) Sarpkaya, I.; Ahmadi, E. D.; Shepard, G. D.; Mistry, K. S.; Blackburn, J. L.; Strauf, S. Strong Acoustic Phonon Localization in Copolymer-Wrapped Carbon Nanotubes. ACS Nano 2015, 9, 63836393.

(54) He, X.; Gifford, B. J.; Hartmann, N. F.; Ihly, R.; Ma, X.; Kilina, S. V.; Luo, Y.; Shayan, K.; Strauf, S.; Blackburn, J. L.; Tretiak, S.; Doorn, S. K.; Htoon, H. Low-Temperature Single Carbon Nanotube Spectroscopy of $\mathrm{sp}^{3}$ Quantum Defects. ACS Nano 2017, 11, 1078510796.

(55) Kwon, H.; Kim, M.; Nutz, M.; Hartmann, N. F.; Perrin, V.; Meany, B.; Hofmann, M. S.; Clark, C. W.; Htoon, H.; Doorn, S. K.; Högele, A.; Wang, Y. Probing Trions at Chemically Tailored Trapping Defects. ACS Cent. Sci. 2019, 5, 1786-1794.

(56) Nutz, M.; Zhang, J.; Kim, M.; Kwon, H.; Wu, X.; Wang, Y.; Högele, A. Photon Correlation Spectroscopy of Luminescent Quantum Defects in Carbon Nanotubes. Nano Lett. 2019, 19, $7078-7084$

(57) Harrah, D. M.; Schneck, J. R.; Green, A. A.; Hersam, M. C.; Ziegler, L. D.; Swan, A. K. Intensity-Dependent Exciton Dynamics of 
(6,5) Single-Walled Carbon Nanotubes: Momentum Selection Rules, Diffusion, and Nonlinear Interactions. ACS Nano 2011, 5, 98989906.

(58) Palotas, J.; Negyedi, M.; Kollarics, S.; Bojtor, A.; Rohringer, P.; Pichler, T.; Simon, F. Incidence of Quantum Confinement on Dark Triplet Excitons in Carbon Nanotubes. ACS Nano 2020, 14, 1125411261.

(59) Schiemann, O.; Cekan, P.; Margraf, D.; Prisner, T. F.; Sigurdsson, S. T. Relative Orientation of Rigid Nitroxides by Peldor: Beyond Distance Measurements in Nucleic Acids. Angew. Chem., Int. Ed. 2009, 48, 3292-3295.

(60) Zhang, Y.; Valley, N.; Brozena, A. H.; Piao, Y.; Song, X.; Schatz, G. C.; Wang, Y. Propagative Sidewall Alkylcarboxylation That Induces Red-Shifted Near-IR Photoluminescence in Single-Walled Carbon Nanotubes. J. Phys. Chem. Lett. 2013, 4, 826-830.

(61) Danné, N.; Kim, M.; Godin, A. G.; Kwon, H.; Gao, Z.; Wu, X.; Hartmann, N. F.; Doorn, S. K.; Lounis, B.; Wang, Y.; Cognet, L. Ultrashort Carbon Nanotubes That Fluoresce Brightly in the NearInfrared. ACS Nano 2018, 12, 6059-6065.

(62) Gao, Z.; Varela, J. A.; Groc, L.; Lounis, B.; Cognet, L. Toward the Suppression of Cellular Toxicity from Single-Walled Carbon Nanotubes. Biomater. Sci. 2016, 4, 230-244.

(63) Marangon, I.; Ménard-Moyon, C.; Kolosnjaj-Tabi, J.; Béoutis, M. L.; Lartigue, L.; Alloyeau, D.; Pach, E.; Ballesteros, B.; Autret, G.; Ninjbadgar, T.; Brougham, D. F.; Bianco, A.; Gazeau, F. Covalent Functionalization of Multi-Walled Carbon Nanotubes with a Gadolinium Chelate for Efficient $\mathrm{T}_{1}$-Weighted Magnetic Resonance Imaging. Adv. Funct. Mater. 2014, 24, 7173-7186.

(64) Rajca, A.; Wang, Y.; Boska, M.; Paletta, J. T.; Olankitwanit, A.; Swanson, M. A.; Mitchell, D. G.; Eaton, S. S.; Eaton, G. R.; Rajca, S. Organic Radical Contrast Agents for Magnetic Resonance Imaging. J. Am. Chem. Soc. 2012, 134, 15724-15727.

(65) Strano, M. S.; Dyke, C. A.; Usrey, M. L.; Barone, P. W.; Allen, M. J.; Shan, H.; Kittrell, C.; Hauge, R. H.; Tour, J. M.; Smalley, R. E. Electronic Structure Control of Single-Walled Carbon Nanotube Functionalization. Science 2003, 301, 1519-1522. 